\title{
Pregnancy after Liver Transplantation
}

\author{
Mohamed Nabih EL Gharib* \\ Department of Obstetrics and Gynecology, Tanta University, Egypt
}

Received: 阱 April 05, 2018; Published: 制 April 17, 2018

*Corresponding author: Mohamed Nabih EL Gharib, Department of Obstetrics and Gynecology, Faculty of Medicine, Tanta University, Egypt

\section{Introduction}

In 1978, Walcott and coworkers documented the first pregnancy in a liver transplant recipient [1]. The recipient's immunosuppressive regimen included prednisone and azathioprine. Her pregnancy was uncomplicated, and she delivered a healthy male, weighing $2400 \mathrm{~g}$, at term. Since Walcott's report, there have been hundreds of pregnancies reported worldwide in liver transplant recipients. Whether the transplant could take in an adverse effect of the pregnancy or whether the pregnancy could potentiate liver transplant it is not settled [2].

\section{Liver Transplant and Timing of Pregnancy}

a) Women should wait at least 1 year after receiving a solidorgan transplant before attempting to get significant, and then should do so but when earned by the transplant team and obstetrician, with close monitoring [3].

b) The best contraceptive method, at least early after liver transplantation, is a barrier method. Later, when the patient is stabilized, hormonal methods may be considered. Sterilization is an option for patients who have completed their family [4].

c) Liver transplant recipients considering pregnancy should have preconception counseling with a maternal-fetal medicine specialist and their transplant physician. There are no specific guidelines for liver transplant recipients to decide whether an allograft is functioning sufficiently well for pregnancy as there is for kidney transplant recipients. Nevertheless, a general assessment of graft function and the status of comorbidities, such as high blood pressure, should be done before conception. The preconceptional visit also provides an opportunity to look back with the patient outcomes of pregnancies with liver transplants, potential effects of immunosuppressive agents on the pregnancy and fetus, and the plan of management for the gestation.

\section{Pregnancy outcome after liver transplantation}

Despite advances in immunosuppressive therapy, pregnancies in liver transplant recipients are still more risky than in the general population for both the mother and the fetus. Pregnancies in liver transplant recipients should be followed up more cautiously by a multidisciplinary team because $[5,6]$ :

a) Overall, $78 \%$ of pregnancies were successful.

b) Many recipients of solid-organ transplants, including liver transplants, develop high blood pressure and renal dysfunction. It is easily grounded in the obstetrical literature that these conditions are independently linked with adverse pregnancy events. The most common obstetric complications were high blood pressure (28\%), preeclampsia $(12 \%)$, and reversible liver dysfunction (27\%) [7].

c) The rate of acute rejection was $10 \%$ [8].

d) The incidence of perinatal and neonatal death was about $4 \%$.

e) Thither was a 3\% incidence of congenital malformations, after exclusion of an infant with the presumed fetal alcohol syndrome and an infant with an autosomal dominant genetic disorder inherited from the female parent.

f) The maternal death rate was $5.5 \%$, over a 2 -year period following delivery [9].

g) Additionally, it seems that the danger of infection during gestation is also significantly increased after liver transplant; this finding may be a consequence of chronic immunosuppressive therapy [1].

h) Liver transplantation does not significantly modify pretransplant diabetes mellitus. Diabetes mellitus frequently develops de novo after liver transplantation, although this complication is usually short-lived and likely linked to immunosuppressive drug administration. The prognosis of patients with post-transplant diabetes mellitus is worse than that of those without this complication [10].

i) The incidence of preterm delivery is $24 \%$ [8]. 
j) The rate of cesarean delivery for liver transplant recipients is higher $(20 \%-100 \%)$ than the national average [11].

k) The rate of newborn complications was also high, at 29\%.

1) Subjective hearing impairment is frequent in patients after OLT and contributes to post-OLT morbidity. Calcineurin inhibitor-related neurotoxicity appears as a potential mechanism. Further prospective investigations with objective hearing tests are necessary to substantiate these results and to assess the use of immunosuppression [12].

\section{Management Recommendations}

a) Patients with liver transplants considering pregnancy should receive preconception counseling. The preconceptional evaluation will require an appraisal of the current graft status and episodes of rejection.

b) Preconceptional counseling should be provided to both the patient and her spouse. For patients who bear a genetic disorder underlying their liver disease, genetic counseling should also be provided. These will include patients with alpha-1antitrypsin deficiency and hemochromatosis, as well as less common disorders such as Alagille syndrome and Caroli syndrome. Prenatal testing may be usable for certain disorders.

c) Ideally, immunosuppressive medications are at stable doses, and no episodes of rejection have occurred in the old yr. An assessment of renal, cardiovascular, and pulmonary status should be performed.

d) Comorbidities such as diabetes and hypertension should be addressed and ideally should be easily-controlled before conception.

e) The status of hepatitis B and C infections as easily as the CMV status should be known and the possible impact on pregnancy discussed. Patients should have up-to-date vaccinations for influenza, pneumococcus, tetanus, and hepatitis B if indicated. In summation, a discussion of pregnancy results in transplant recipients should take place, concentrating especially on the potential for and long-term outcomes of preterm parturition.

f) Pregnancies in patients with liver transplants should be done in an interdisciplinary fashion between a maternal-fetal medicine specialist, a transplant physician, and other specialists as indicated.

g) Transplant recipients will require frequent prenatal visits throughout the class of their pregnancies.

h) Close monitoring of line pressure, proteinuria, and laboratory studies throughout the pregnancy are all-important.

i) Patients on chronic prednisone should have early screening for gestational diabetes. Although the frequency of congenital birth defects does not appear to be increased with immunosuppressive agents in studies thus far, a genetic sonogram is indicated for transplant patients to boot out the presence of congenital abnormalities.

j) Serial growth scans should be done throughout pregnancy to monitor for fetal growth restriction.

k) In the third trimester, antenatal testing may be done, although the benefit of testing in this population has not been established.

l) Cesarean delivery should be performed for standard obstetrical indications.

m) Stress-dose steroids are shown at the time of delivery for patients who have been taking corticosteroids during pregnancy.

n) An increase in baseline liver function needs an aggressive evaluation in these pregnancies. On that point is no contraindication to liver biopsy in these patients to evaluate for graft rejection.

o) Several other complications of pregnancy, such as preeclampsia, HELLP syndrome, and cholestasis of pregnancy, may result in elevations of liver enzymes. Some small reports have demonstrated an increase in the rate of cholestasis of pregnancy in liver transplant recipients, but this has not been measured in larger works. It may be rather challenging to identify these complications of pregnancy from graft rejection or exacerbation of the underlying liver disease, such as hepatitis C.

p) The goal of managing these pregnancies is to optimize maternal health, including graft function and co morbidities, to find and manage complications of pregnancy such as preeclampsia and infection, and to downplay the complications of preterm birth whenever possible.

q) From the literature, it appears that many liver transplant recipients can look at safe gestation and neonatal outcomes. However, at that place is a big deal of research needed to clarify the optimal management of these pregnancies.

\section{References}

1. Walcott WO, Derick DE, Jolley JJ, Snyder D (1978) Successful pregnancy in a liver transplant patient. Am J Obstet Gynecol 132 (3): 340-341.

2. Women in Hepatology Group, Italian Association for the Study of the Liver (AISF) (2016) AISF position paper on liver transplantation and pregnancy: Women in Hepatology Group, Italian Association for the Study of the Liver (AISF). Dig Liver Dis 48(8): 860-868.

3. Ramirez CB, Doria C (2014) Pregnancy after liver transplantation. Best Pract Res Clin Obstet Gynaecol 28(8): 1137-1145.

4. Riely AC (2001) Contraception and Pregnancy After Liver Transplantation. Liver Transplantation 7(11): S74-S76. 
5. Baskiran A, Karakas S, Ince V, Kement M, Ozdemir F, et al. (2017) Pregnancy After Liver Transplantation: Risks and Outcomes. Transplant Proc 49(8): 1875-1878.

6. Coscia LA, Constantinescu S, Moritz MJ, Frank AM, Ramirez CB, et al (2010) Report from the National Transplantation Pregnancy Registry (NTPR): outcomes of pregnancy after transplantation. Clin Transpl pp. 65-85.

7. Brosens I, Brosens JJ, Benagiano G (2014) The risk of obstetrical syndromes after solid organ transplantation. Best Pract Res Clin Obstet Gynaecol 28: 1211-1221.

8. Mattila M, Kemppainen H, Ispniemi H, Kantola (2017) Pregnancy outcomes after liver transplantation in Finland. AOGS 96(9): 1106-1111.
9. Gelson W, Hoare M, Dawwas MF, Gibbs P, Alexander G, et al. (2011) The pattern of late mortality in liver transplant recipients in the United Kingdom. Transplantation 91: 1240-1244.

10. Navasa M, Bustamante J, Marroni C, González E, Andreu H, et al. (1996) Diabetes mellitus after liver transplantation: prevalence and predictive factors. J Hepatol 25(1): 64-71.

11. Coffin CS, Shaheen AA, Burak KW, Myers RP (2010) Pregnancy outcomes among liver transplant recipients in the United States: a nationwide case-control analysis. Liver Transpl 16(1): 56-63.

12. Rifai K, Kirchner GI, Bahr MJ, Cantz T, Rosenau J, et al. (2006) A new side effect of immunosuppression: high incidence of hearing impairment after liver transplantation. Liver Transpl 12(3): 411-415.

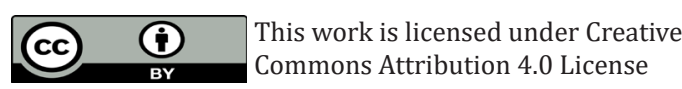

To Submit Your Article Click Here:

Submit Article

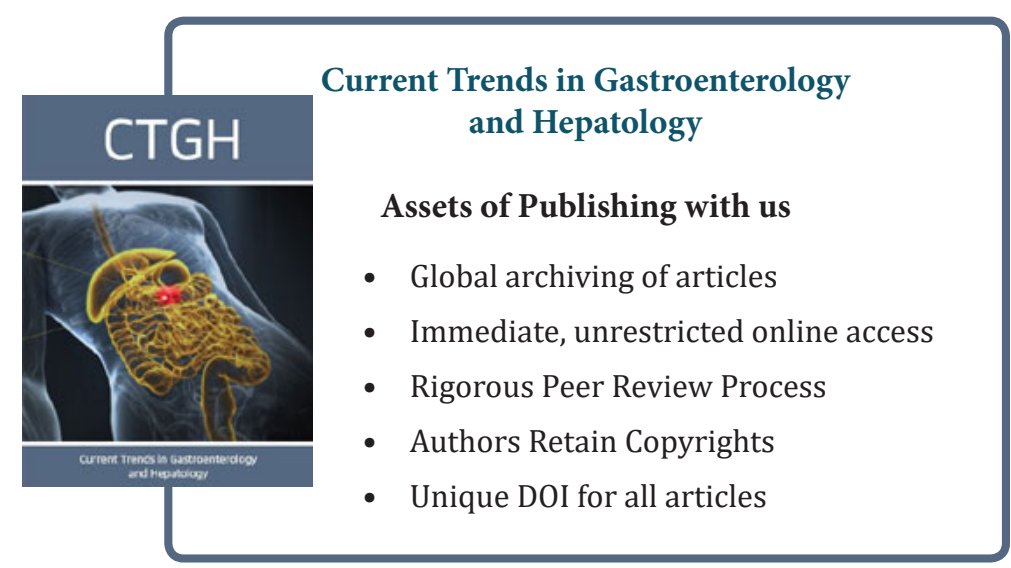

